\title{
Spatially-Aware Tangibles Using Mouse Sensors
}

\author{
Dennis Schüsselbauer \\ University of Regensburg \\ Regensburg, Germany \\ dennis.schuesselbauer@stud.uni-regensburg.de \\ Raphael Wimmer \\ University of Regensburg \\ Regensburg, Germany \\ raphael.wimmer@ur.de
}

\author{
Andreas Schmid \\ University of Regensburg \\ Regensburg, Germany \\ andreas1.schmid@stud.uni-regensburg.de \\ Laurin Muth \\ University of Regensburg \\ Regensburg, Germany \\ laurin.muth@stud.uni-regensburg.de
}

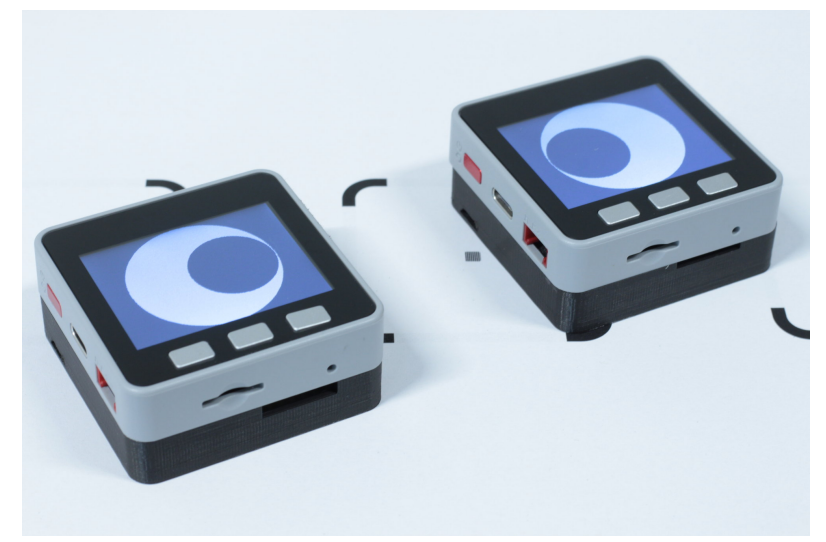

Figure 1: Prototypes of a self-tracking tangible based on an M5Stack block and a PMW3360DM optical mouse sensor. The raw image captured by the sensor can be used for determining the absolute location while the displacement reported by the sensor firmware allows for robustly tracking the tangibles' positions.

ad-hoc wireless network. The demo allows users to evaluate the properties of our current implementation and explore applications supported by self-tracking tangibles. We present the following exemplary applications: value selection by dragging a physical slider, a magic lens for exploring large images, and two tangibles tracking each other's position (Figure 1).

\section{ACKNOWLEDGMENTS}

This project is funded by the Bavarian State Ministry of Education, Science and the Arts in the framework of the Centre Digitisation.Bavaria (ZD.B).

\section{REFERENCES}

1. Dennis Schüsselbauer, Andreas Schmid, Raphael Wimmer, and Laurin Muth. 2018. DotTrack: absolute und relative Ortsbestimmung von Tangibles mittels eines Maussensors. In Mensch und Computer 2018 - Workshopband, 183-188. https://doi.org/10.18420/muc2018-ws05-0500 